\title{
A Theoretical Framework for Selecting the Cost Function for Source Routing
}

\author{
Gang Cheng and Nirwan Ansari, Senior Member, IEEE
}

\begin{abstract}
Finding a feasible path subject to multiple constraints in a network is an NP-complete problem and has been extensively studied. Many proposed source routing algorithms tackle this problem by transforming it into the shortest path selection problem, which is $\mathbf{P}$-complete, with an integrated cost function that maps the multi-constraints of each link into a single cost. However, how to select an appropriate cost function is an important issue that has rarely been addressed in literature. In this paper, we provide a theoretical framework for picking a cost function that can improve the performance of source routing in terms of complexity, convergence, and probability of finding a feasible path.
\end{abstract}

Index Terms - Multiple additively constrained QoS routing, cost function, NP-complete.

\section{INTRODUCTION}

$\mathrm{T}$ he tremendous growth of the global Internet has given rise to a variety of applications that require quality-of-service (QoS) beyond what is provided by the current best-effort IP packet delivery service. One of the challenging issues is to select feasible paths that satisfy different quality-of-service requirements. This problem is known as QoS routing. In general, state distribution and routing strategy [1] are the two issues related to QoS routing. State distribution addresses the issue of exchanging the state information throughout the network [2]. Routing strategy is used to find a feasible path that meets the QoS requirements. In this paper, we focus on the latter task, and assume that accurate network state information is available to each node. A number of research works have also addressed inaccurate information [3, 4], which is, however, beyond the scope of this paper.

QoS constraints can be categorized into three types: concave, additive, and multiplicative. Since concave parameters set the upper limits of all the links along a path such as bandwidth, we can simply prune all the links and nodes that do not satisfy the QoS constraints. We can also convert multiplicative parameters into additive parameters by using the logarithm function. For instance, we can take $-\log (1-p)$ as the replacement for loss rate $p$. Thus, we focus only on additive constraints in this paper. It has been proved that multiple

Authors are with the Advanced Networking Laboratory, Newark, NJ 07012, U.S.A. (corresponding author to provide phone: 973-596-3670; fax: 973-596-5680; e-mail: ansari@njit.edu).

This work has been supported in part by the New Jersey Commission on Higher Education via the NJ I-TOWER project, and the New Jersey Commission on Science and Technology via the NJ Center for Wireless Telecommunications. additively constrained QoS routing is NP-complete [5]. Hence, tackling this problem requires heuristics. In [6], a heuristic algorithm was proposed based on a linear cost function for two additive constraints; this is a MCP (Multiple Constrained Path Selection) [1] problem with two additive constraints. A binary search strategy for finding the appropriate value of $k$ in the linear cost function $w_{1}(p)+k w_{2}(p)$ or $k w_{1}(p)+w_{2}(p)$, where $w_{i}(p)(i=1,2)$ are two respective weights of the path $p$, was proposed, and a hierarchical Dijkstra algorithm was introduced to find the path. It was shown that the worst-case complexity of the algorithm is $\mathrm{O}(\log B(m+n \log n))$, where $B$ is the upper bound of the parameter $k, m$ is the number of links, and $n$ is the number of nodes. Similar to [6], Lagrange Relaxation Based Aggregated Cost (LARAC) was proposed in [7] for the Delay Constrained Least Cost path problem $(D C L C)$. This algorithm is based on a linear cost function $c_{\lambda}=c+\lambda d$, where $c$ denotes the cost, $d$ the delay, and $\lambda$ an adjustable parameter. It differs from [6] on how $\lambda$ is defined: $\lambda$ is computed by Lagrange Relaxation instead of the binary search. It was shown that the computational complexity of this algorithm was $\mathrm{O}\left(m^{2} \log ^{4} m\right)$. However, in [8], for the same problem $(D C L C)$, a non-linear cost function was proposed after considering the shortcoming of the linear cost function.

Many proposed source routing algorithms transform the multiple constrained QoS routing problem into a shortest path selection problem with an integrated cost function that map the multi-constraints of each link into a single cost. However, how to select an appropriate cost function is rarely addressed in literature; hereby we will provide a theoretical framework for this issue.

\section{PROBLEM ForMULATION}

We will provide a general framework for selecting a cost function in which there is no limitation on the number of QoS constraints. Since concave constraints can be easily addressed by pruning, and multiplicative constraints can be generally converted into additive constraints, without loss of generality, we only consider additive constraints and formulate the problem as follows:

Definition 1: Multiple Additively Constrained Path Selection $(M A C P)$ : Assume a network is modeled as a directed graph $G(N, E)$, where $N$ is the set of all nodes and $E$ is the set of all links. Each link connected from node $u$ to $v$, denoted by $e_{u, v}=(u, v) \in E$, is associated with $M$ additive parameters: 
$w_{i}(u, v) \geq 0, \quad i=1,2, \ldots, M$. Given a set of constraints $\left(c_{1}, c_{2}, \ldots, c_{M}\right)$ and a pair of nodes $s$ and $t$, MACP is to find a path $p$ from $s$ to $t$ subject to $W_{i}(p)=\sum_{e_{u, v} \in p} w_{i}(u, v)<c_{i}$, for all $i=1,2, \ldots, M$.

Definition 2: Any path selected by MACP is a feasible path; that is, any path $p$ from $s$ to $t$ that meets the requirement, $W_{i}(p)=\sum_{e_{u, v} \in p} w_{i}(u, v) \leq c_{i}$, for all $i=1,2, \ldots, M$, is a feasible path.

\section{Notations:}

1. $f(\underline{\mathbf{x}})$ : Cost function, where $\underline{\mathbf{x}}=\left(x_{1}, x_{2}, \ldots, x_{M}\right)$.

2. $\mathbf{C}:$ The vector representation of the QoS constraints $\left(c_{1}, c_{2}, \ldots, c_{M}\right)$.

3. $\mathbf{W}(p)$ : The weight vector of path $p$, i.e., $\underline{\mathbf{W}(p)}=\left(W_{1}(p), W_{2}(p), \ldots, W_{M}(p)\right)$, where $W_{i}(p)=$ $\sum_{e_{u, v} \in p} w_{i}(u, v)$.

4. $C(p)$ : The cost of path $p$, $C(p)=\sum_{e_{u, v} \in p} f\left(w_{1}\left(e_{u, v}\right), w_{2}\left(e_{u, v}\right), \ldots, w_{M}\left(e_{u, v}\right)\right), \quad$ where

$f(\cdot)$ is the cost function.

Note that $C(p) \neq f(\mathbf{W}(p))$ for

$$
f(\underline{\mathbf{W}(p)})=f\left(\sum_{e_{u, v} \in p} w_{1}(u, v), \sum_{e_{u, v} \in p} w_{2}(u, v), \ldots, \sum_{e_{u, v} \in p} w_{M}(u, v)\right) .
$$

However, if $f(\underline{\mathbf{x}})$ is linear, i.e., $f(\underline{\mathbf{x}})=\sum_{i=1}^{M} \beta_{i} x_{i}$,

$$
\begin{aligned}
& f(\underline{\mathbf{W}}(p))=f\left(\sum_{e_{u, v} \in p} w_{1}(u, v), \sum_{e_{u, v} \in p} w_{2}(u, v), \ldots, \sum_{e_{u, v} \in p} w_{M}(u, v)\right) \\
&= \sum_{i=1}^{M} \beta_{i}\left(\sum_{e_{u, v} \in p} w_{i}(u, v)\right) \\
&=\left.\sum_{i=1}^{M} \sum_{e_{u, v} \in p} \beta w_{i}(u, v)\right) \\
&= \sum_{e_{u, v} \in p}\left(\sum_{i=1}^{M} \beta_{i} w_{i}(u, v)\right) \\
&=\sum_{e_{u, v} \in p} f\left(w_{1}\left(e_{u, v}\right), w_{2}\left(e_{u, v}\right), \ldots, w_{M}\left(e_{u, v}\right)\right)=C(p)
\end{aligned}
$$

\section{A FRAMEWORK FOR SELECTING THE COST FunCTION}

Many proposed source routing algorithms are associated with cost functions, which are essential for solving the problem. Thus, designing an appropriate cost function is a key issue in this kind of approaches. In this paper, by assuming the cost function is continuous, we present some basic features of the cost function and the impact of these features on the performance of routing algorithms.

Without loss of generality, the cost function for traversing a link from node $u$ to $v$ is desirable to have the following properties:

1. $f(0,0, \ldots, 0)=0$; each variable in $f(\cdot)$ corresponds to a QoS parameter such as delay and jitter. It is intuitive that the cost for traversing a link with " 0 " QoS value (e.g., the cost of traversing a link which does not cause any delay) should be " 0 ".
2. $\frac{\partial f(\underline{\mathbf{x}})}{\partial x_{i}}>0$ if $x_{i}>0$ and $\frac{\partial f(\underline{\mathbf{x}})}{\partial x_{i}} \geq 0 \quad$ if $\quad x_{i}=0$, $i=1,2, \ldots, M$; i.e., the cost function is increasing with respect to each additive parameter.

3. $\frac{\partial^{2} f\left(x_{1}, x_{2}, \ldots, x_{M}\right)}{\partial x_{i}^{2}} \geq 0$; i.e., the cost function is concave implying that if $\sum_{e_{u, v} \in p} w_{i}(u, v) \leq c_{i}$, $i=1,2, \ldots, M$, then $C(p) \leq f(\underline{\mathbf{W}(p))} \leq f(\underline{C})$. In fact, most source routing algorithms proposed in the literature possess this property.

Note that some other non-continuous functions proposed for QoS routing can also be characterized by functions which satisfy the above properties. For example, $f\left(x_{1}, x_{2}\right)=\max \left\{x_{1}, x_{2}\right\} \quad$ equals to $\lim _{n \rightarrow \infty}\left(x_{1}^{n}+x_{2}^{n}\right)^{1 / n}$ which satisfies the above properties. The following theorems and lemmas are derived by assuming that the cost function $f(\underline{\mathbf{x}})$ satisfies Properties 1-3.

[Theorem 1] No feasible path exists if the least cost path has the cost larger than $f(\underline{\mathbf{C}})$.

Proof: By contradiction. Assume path $\hat{p}$ satisfies the constraint $\underline{\mathbf{C}}$ and the least cost among all paths is larger than $f(\underline{\mathbf{C}})$; that is,

$$
C(p)>f(\underline{\mathbf{C}}), \forall p \Rightarrow C(\hat{p})>f(\underline{\mathbf{C}}) .
$$

Also

$$
\frac{\partial^{2} f\left(x_{1}, x_{2}, \ldots, x_{M}\right)}{\partial x_{i}^{2}} \geq 0 \Rightarrow f(\underline{\mathbf{W}(\hat{p})}) \geq C(\hat{p}) .
$$

Thus, from (3) and (4),

$$
f(\underline{\mathbf{W}(\hat{p}))}>f(\underline{\mathbf{C}}) .
$$

However, since $\frac{\partial f(\underline{\mathbf{x}})}{\partial x_{i}} \geq 0$ and path $\hat{p}$ satisfies the constraint $\underline{\mathbf{C}}$,

$$
W_{i}(\hat{p}) \leq c_{i}, \forall i \in\{1,2, \ldots, M\} \Rightarrow f(\underline{\mathbf{W}(\hat{p})}) \leq f(\underline{\mathbf{C}}),
$$

which contradicts (5), and thus Theorem 1 is proved.

The following lemma will facilitate the proof of Theorem 2 .

[Lemma 1] Let $g_{i}(x)=f\left(x \overrightarrow{\mathbf{e}}_{\mathbf{i}}\right)$, where $\overrightarrow{\mathbf{e}}_{\mathbf{i}}$ is the $i$ th unit vector in the $m$-dimensional space, and $\sum_{m=1}^{K} g_{i}\left(y_{m}\right)=\delta, y_{i} \geq 0$, $i=1,2, \ldots, K$. Then:

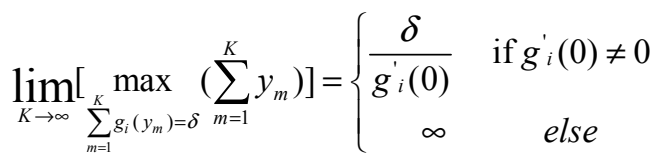

where $g_{i}^{\prime}(0)=\left.\frac{d g_{i}(x)}{d x}\right|_{x=0}=\left.\frac{\partial f(\underline{\mathbf{x}})}{\partial x_{i}}\right|_{\underline{\mathbf{x}}=\underline{\mathbf{0}}}$.

Proof: Let

$$
L\left(y_{1}, y_{2}, \ldots, y_{K}, \lambda\right)=\sum_{m=1}^{K} y_{m}-\lambda\left[\sum_{m=1}^{K} g_{i}\left(y_{m}\right)-\delta\right] .
$$

Thus 


$$
\begin{aligned}
& \frac{d L\left(y_{1}, y_{2}, \ldots, y_{K}, \lambda\right)}{d y_{j}}=0 \Rightarrow 1-\lambda g_{i}^{\prime}\left(y_{j}\right)=0 \\
& \Rightarrow g_{i}^{\prime}\left(y_{j}\right)=\frac{1}{\lambda}, j=1,2, \ldots, K .
\end{aligned}
$$

By letting $y_{1}=y_{2}=\ldots=y_{K}$,

$$
g_{i}\left(y_{m}\right)=\frac{\delta}{K}, m=1,2, \ldots, K .
$$

Also

$$
g_{i}^{\prime \prime}(x) \geq 0 \Rightarrow \frac{\partial^{2} L\left(y_{1}, y_{2}, \ldots, y_{K}, \lambda\right)}{\partial y_{j}^{2}} \leq 0, j=1,2, \ldots, K .
$$

Thus, $\sum_{m=1}^{K} y_{m}$ is maximized by (9).

Define $I_{i}(x)$ as the inverse function of $g_{i}(x)$. So,

$$
\max \left(\sum_{m=1}^{K} y_{m}\right)=\sum_{m=1}^{K} I_{i}\left(\frac{\delta}{K}\right)=K I_{i}\left(\frac{\delta}{K}\right) .
$$

Since $g_{i}^{\prime \prime}(x) \geq 0, \quad g_{i}^{\prime}(x) \geq 0 \quad$ and $\quad g_{i}(0)=0$, we have $\frac{d I_{i}(x)}{d x} \geq 0$ and $\frac{d^{2} I_{i}(x)}{d x^{2}} \leq 0$. Hence, $\max \left(\sum_{m=1}^{K} y_{m}\right)$ increases with $K$. Thus,

$$
\begin{aligned}
& \lim _{K \rightarrow \infty}\left[\max \left(\sum_{m=1}^{K} y_{i}\right)\right]=\lim _{K \rightarrow \infty}\left[K I_{i}\left(\frac{\delta}{K}\right)\right]=\lim _{K \rightarrow \infty}\left[\delta \frac{I_{i}\left(\frac{\delta}{K}\right)}{\frac{\delta}{K}}\right] \\
& =\delta \lim _{x \rightarrow 0} \frac{I_{i}(x)}{x}=\left\{\begin{array}{cc}
\frac{\delta}{g_{i}^{\prime}(0)} & \text { if } g_{i}^{\prime}(0) \neq 0 \\
\infty & \text { else }
\end{array}\right.
\end{aligned}
$$

[Theorem 2] A path $p$ is a feasible path if $C(p) \leq \min _{i}\left\{\left.c_{i} \frac{\partial f(\underline{\mathbf{x}})}{\partial x_{i}}\right|_{\underline{\mathbf{x}}=\underline{0}}\right\}$.

Proof: Since $w_{m}\left(e_{u, v}\right) \geq 0, m=1,2, \ldots, M$,

$$
g_{i}\left(w_{i}\left(e_{u, v}\right)\right) \leq f\left(\underline{\mathbf{W}\left(e_{u, v}\right)}\right), i=1,2, \ldots, M .
$$

So, for any path $p$,

$$
\sum_{e_{u, v} \in p} g_{i}\left(w_{i}\left(e_{u, v}\right)\right) \leq C(p), i=1,2, \ldots, M .
$$

Then, for a path $p$ with $C(p) \leq \min _{i}\left\{\left.c_{i} \frac{\partial f(\underline{\mathbf{x}})}{\partial x_{i}}\right|_{\underline{\mathbf{x}}=\underline{0}}\right\}$,

$$
\sum_{e_{u, k} \in p} g_{i}\left(w_{i}\left(e_{u, v}\right)\right) \leq C(p) \leq \min _{i}\left\{\left.c_{i} \frac{\partial f(\underline{\mathbf{x}})}{\partial x_{i}}\right|_{\underline{\mathbf{x}}=\underline{0}}\right\}, i=1,2, \ldots, M .
$$

Consider the case that if $\left.\frac{\partial f(\underline{\mathbf{x}})}{\partial x_{i}}\right|_{\underline{\mathbf{x}} \mathbf{0}} \neq 0 \quad i=1,2, \ldots, M$, by Lemma 1,

$$
\sum_{e_{u, v} \in p} w_{i}\left(e_{u, v}\right) \leq \frac{\min _{i}\left\{\left.c_{i} \frac{\partial f(\underline{\mathbf{x}})}{\partial x_{i}}\right|_{\underline{\mathbf{x}}=\mathbf{0}}\right\}}{\left.\frac{\partial f(\underline{\mathbf{x}})}{\partial x_{i}}\right|_{\underline{\mathbf{x}}=\underline{0}}} \leq \frac{\left.c_{i} \frac{\partial f(\underline{\mathbf{x}})}{\partial x_{i}}\right|_{\underline{\mathbf{x}}=\underline{0}}}{\left.\frac{\partial f(\underline{\mathbf{x}})}{\partial x_{i}}\right|_{\underline{\underline{x}} \underline{\mathbf{0}}}}=c_{i} .
$$

Consider the other case that $\left.\exists i \in\{1,2, \ldots, M\} \ni \frac{\partial f(\underline{\mathbf{x}})}{\partial x_{i}}\right|_{\underline{\mathbf{x}}=\underline{0}}=0$. Thus, $\min _{i}\left\{\left.c_{i} \frac{\partial f(\underline{\mathbf{x}})}{\partial x_{i}}\right|_{\underline{\mathbf{x}}=\underline{0}}\right\}=0 \Rightarrow C(p)=0 \Rightarrow w_{i}\left(e_{u, v}\right)=0, \forall e_{u, v} \in p$

Thus

$$
W_{i}(p)=\sum_{e_{u, u}, p} w_{i}\left(e_{u, v}\right)=0 \leq c_{i}, \forall i \in\{1,2, \ldots, M\} .
$$

That is, path $p$ is a feasible path.

Note that a path $p$ with $C(p) \leq f(\underline{\mathbf{C}})$ may not be a feasible path. For example, consider $f\left(x_{1}, x_{2}\right)=x_{1}+x_{2}$, with $\underline{\mathbf{C}}=(1,1)$, and a path $p$ with $\mathbf{W}(p)=(1.1,0)$. It is obvious that $C(p)=f(\underline{\mathbf{W}(p))}<f(\underline{\mathbf{C}})$, but path $p$ is not a feasible path.

The following two definitions can be used as the stopping criteria in searching for feasible paths.

Definition 3: Given a network $G$, with cost function $f$ and QoS constraint $\underline{\mathbf{C}}$, no feasible path exists from node $s$ to $t$ if the least cost path $p$ from node $s$ to $t$ has $C(p)>B_{u}(f, G, \underline{\mathbf{C}}) . \quad B_{u}(f, G, \underline{\mathbf{C}})$ is known as an upper bound of the cost of a feasible path.

Definition 4: Given a network $G$, with cost function $f$, and QoS constraint $\underline{\mathbf{C}}$, if any path $p$ from node $s$ to $t$ with $C(p) \leq B_{t}(f, G, \underline{\mathbf{C}})$ is a feasible path, $B_{t}(f, G, \underline{\mathbf{C}})$ is known as the tight bound of the cost of a feasible path.

From Definitions 3 and 4, the cost of a feasible path cannot be larger than $B_{u}(f, G, \underline{\mathbf{C}})$, and that of an infeasible path is larger than $B_{t}(f, G, \underline{\mathbf{C}})$. It also follows from Theorems 1 and 2 that $B_{u}(f, G, \underline{\mathbf{C}}) \leq f(\underline{\mathbf{C}})$ and $B_{t}(f, G, \underline{\mathbf{C}}) \geq \min _{i}\left\{\left.c_{i} \frac{\partial f(\underline{\mathbf{x}})}{\partial x_{i}}\right|_{\underline{\mathbf{x}}=\underline{\underline{0}}}\right\}$.

[Theorem 3] If $\lambda_{i, j}=\min _{u, v \in N}\left\{\frac{w_{i}(u, v)}{w_{j}(u, v)}\right\}$, then $\lambda_{i, j} \leq \frac{W_{i}(p)}{W_{j}(p)}$ for any path $p$.

Proof: Since $\lambda_{i, j}=\min _{u, v \in N}\left\{\frac{w_{i}(u, v)}{w_{j}(u, v)}\right\}$, we have

$$
\lambda_{i, j} w_{j}(u, v) \leq w_{i}(u, v) .
$$

Then,

$$
\frac{W_{i}(p)}{W_{j}(p)}=\frac{\sum_{(u, v) \in p} w_{i}(u, v)}{\sum_{(u, v \in p} w_{j}(u, v)} \geq \frac{\sum_{(u, v) \in p} \lambda_{i, j} w_{j}(u, v)}{\sum_{(u, v) \in p} w_{j}(u, v)}=\lambda_{i, j} .
$$

[Lemma 2] If $\lambda_{i, j}=\min _{u, v \in N}\left\{\frac{w_{i}(u, v)}{w_{j}(u, v)}\right\}$, and $\frac{c_{i}}{c_{j}}<\lambda_{i, j}$ for $1 \leq i, j \leq M$, any path $p$ with $W_{i}(p) \leq c_{i}$ also implies that $W_{j}(p)<c_{j}$.

Proof: From Theorem 3,

$$
\lambda_{i, j} \leq \frac{W_{i}(p)}{W_{j}(p)} \Rightarrow W_{j}(p) \leq \frac{W_{i}(p)}{\lambda_{i, j}} \leq \frac{c_{i}}{\lambda_{i, j}}<c_{j} .
$$

Lemma 2 implies that if $\frac{c_{i}}{c_{j}}<\lambda_{i, j}$, the $j$ th constraint is met as long as the $i$ th constraint is met. Thus, the $j$ th constraint 
can be omitted for the MACP problem; i.e., the $M$-constrained problem can be reduced to an (M-1)-constrained problem.

[Lemma 3] Define $\lambda_{i, j}=\min _{u, v \in N}\left\{\frac{w_{i}(u, v)}{w_{j}(u, v)}\right\} . \quad B_{t}(f, G, \underline{\mathbf{C}}) \geq$ $\min _{i}\left\{\left.\frac{c_{i}}{h_{i}^{\prime}(x)}\right|_{x=0}\right\}$, where $h_{i}(x)=f\left(\lambda_{1, i} x, \lambda_{2, i} x, \ldots, \lambda_{M, i} x\right)$.

Proof: The proof is similar to that of Theorem 2.

[Lemma 4] If $\lambda_{i, j}=\min _{u, v \in N}\left\{\frac{w_{i}(u, v)}{w_{j}(u, v)}\right\}$ and there are $N$ nodes in the network, $B_{t}(f, G, \underline{\mathbf{C}}) \geq \min _{i}\left\{(N-1) \quad h_{i}\left(\frac{c_{i}}{N-1}\right)\right\}$, where $h_{i}(x)=f\left(\lambda_{1, i} x, \lambda_{2, i} x, \ldots, \lambda_{M, i} x\right)$.

Proof: The proof is similar to that of Theorem 2.

[Lemma 5] Given a cost function $f$ and QoS constraint $\underline{\mathbf{C}}$, an $n$-hop path $p$ satisfying $C(p) \leq \min _{i}\left\{n h_{i}\left(\frac{c_{i}}{n}\right)\right\}$ is a feasible path.

Proof: The proof is similar to that of Theorem 2.

is defined as the $n$-hop tight bound; that is, the cost of any $n$-hop infeasible path cannot be less than this bound.

From Theorem 2 and Lemma 5, it is known that an $n$-hop path with cost between the upper bound and $n$-hop tight bound may be an infeasible path. Intuitively, it is desirable to have the upper bound and tight bound close to each other to minimize the possibility of selecting a wrong path. Concatenate $\underline{\mathbf{W}_{1}}, \underline{\mathbf{W}_{2}}, \ldots, \underline{\mathbf{W}_{\mathbf{n}}}$ of links of an $n$-hop path into an $n \cdot M \times 1$ vector $\left(\underline{\mathbf{W}_{1}}, \underline{\mathbf{W}_{2}}, \ldots, \underline{\mathbf{W}_{\mathbf{n}}}\right)$. Thus, the area covered by the $n$-hop tight bound is

$$
A_{t}(f, n)=\int_{\sum_{i=1}^{n} f\left(\underline{\mathbf{W}_{\mathbf{i}}}\right) \leq \min _{i}\left\{n h_{i}\left(\frac{c_{i}}{n}\right)\right\}, \underline{\mathbf{W}_{\mathbf{i}} \leq \underline{\mathbf{C}}, i=1,2, \ldots, M}} \ldots \int d \underline{\mathbf{W}_{\mathbf{1}}} d \underline{\mathbf{W}_{\mathbf{2}} \ldots d \underline{\mathbf{W}_{\mathbf{n}}}} .
$$

Similarly, the area covered by the upper bound is

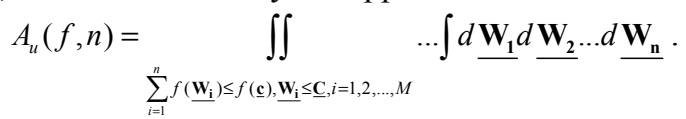

Based on the above intuition, the coverage ratio defined below

$$
r(f, n)=\frac{A_{t}(f, n)}{A_{u}(f, n)}
$$

can be used as a figure of merit on how robust the cost function is in avoiding a "wrong path selection." The larger the ratio, the better the cost function.

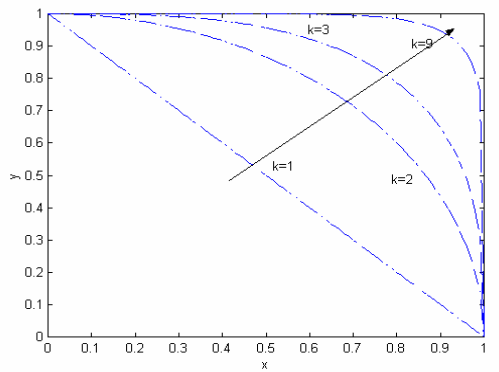

Figure 1. $A_{t}(f, 1)$ for $x^{k}+y^{k}$
For example, let $n=1, \underline{\mathbf{C}}=(1,1), \lambda_{1,2}=\lambda_{2,1}=0$, and the cost function $f(x, y)=x^{k}+y^{k}, k \geq 1$. In this case, $A_{u}(f, n)=1$, and Fig. 1 shows the area covered by the tight bound increases with $k$. Thus, $r(f, n)=1$ as $k \rightarrow \infty$; i.e., an infeasible will not be selected.

However, note that $\min _{i}\left\{n h_{i}\left(\frac{c_{i}}{n}\right)\right\}$ does not change with $n$ if the cost function is linear while $\min _{i}\left\{n h_{i}\left(\frac{c_{i}}{n}\right)\right\}$ decreases with $n$ if $\frac{\partial^{2} f\left(x_{1}, x_{2}, \ldots x_{M}\right)}{\partial x_{i}^{2}}>0, i=1,2, \ldots, M$. Thus, we conjecture that, for any nonlinear cost function, there must exist a linear cost function with a larger $r(f, n)$ when $n$ is large enough. With $\underline{\mathbf{C}}=(1,1), \lambda_{1,2}=\lambda_{2,1}=0$, Fig. 2 and Fig. 3 show the coverage ratios for both $f(x, y)=x^{k}+y^{k}$ and $f 2(x, y)=\left(e^{(k-1) x}+e^{(k-1) y}-2\right) /(k-1), \quad k \geq 1$ when $n=2$ and $n=3$.

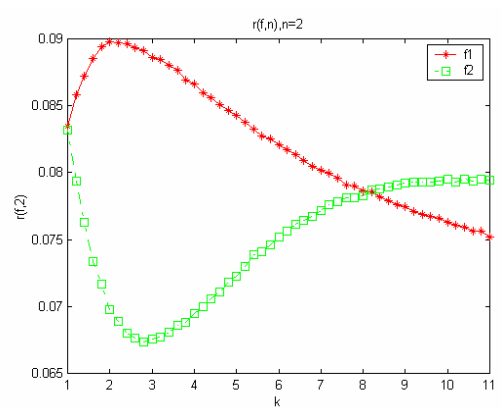

Figure 2. Coverage Ratio for $\mathbf{n}=\mathbf{2}$.

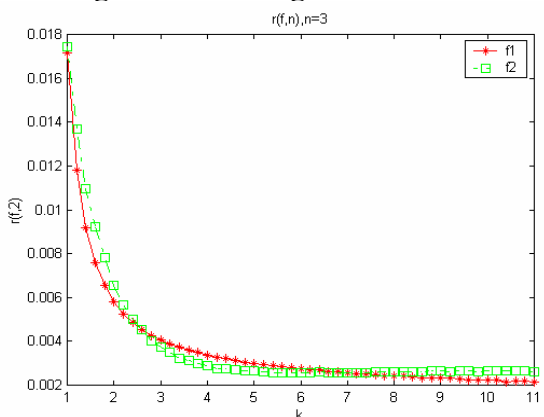

Figure 3. Coverage Ratio for $n=3$.

When $n=3$, the linear cost function (i.e., $x+y$ when $k=0$ ) yields the largest coverage ratio as compared to $f 1(\cdot, \cdot)$ and $f 2(\cdot, \cdot)$. Furthermore, the linear cost function possesses a distinguished property, $C(p)=f(\underline{\mathbf{W}(p)})$, that makes the design of QoS routing algorithm (selection of cost function parameters) such as [6] straightforward and easy. Thus, the linear cost function is preferred for source QoS routing algorithms. This conclusion assumes that the cost of a path is the sum of the costs of all individual links comprising the path; it may not be feasible for algorithms in which the assumption does not hold. For example, a good approach proposed in [10] is that the cost of path $p$ is $f(\underline{\mathbf{W}(p))}$, i.e., $C(p)=f(\underline{\mathbf{W}(p))}$, 
where $f(\cdot)$ is the cost function. In this case, path $p$ can always be viewed as a link connecting the source and the destination with weight $\mathbf{W}(p)$. Hence, if there are only two constraints, and $f(x, y)=x^{k}+y^{k}, k \geq 1$, the success ratio of a finding feasible path using this algorithm always increases with $k$, regardless the number of the hop count from the source to the destination, as shown in Fig. 1. So, in this case, the linear cost function is not optimal, and our conclusion is not applicable anymore.

\section{SIMULATIONS}

We conduct our simulations in two network topologies: one is the network topology presented in [6], [10], and the other is a $7 \times 7$ mesh network. The cost functions adopted for comparisons are $f 1(x, y)=x+y, f 2(x, y)=x^{2}+y^{2}$, and $f 3(x, y)=\left(e^{x}+e^{y}-2\right)$. The QoS routing algorithms used in simulations are Dijkstra algorithm. In both simulations, the link weights are independent and uniformly distributed from 0 to 1, two QoS constraints are set to be equal, and increase from 0.5 to 5.1 with an increment of 0.2 . All data are obtained by running $1,000,000$ requests. To truly reflect algorithms' capability in finding a feasible path, we propose the following more appropriate success ratio definition as our performance index:

$$
S R=\frac{\text { Total number of success request of the algorithm }}{\text { Total number of success request of the optimal algorithm }} .
$$

The algorithm that can always locate a feasible path as long as it exists is refereed to as the optimal algorithm. Here, it is achieved simply by flooding which is rather exhaustive. The simulation results are shown in Figures 4 and 5 .

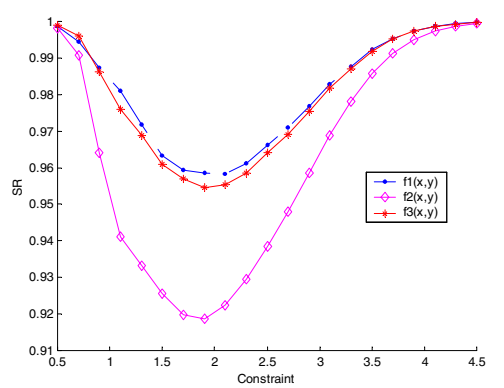

Figure 4. SR in the 32-node network.

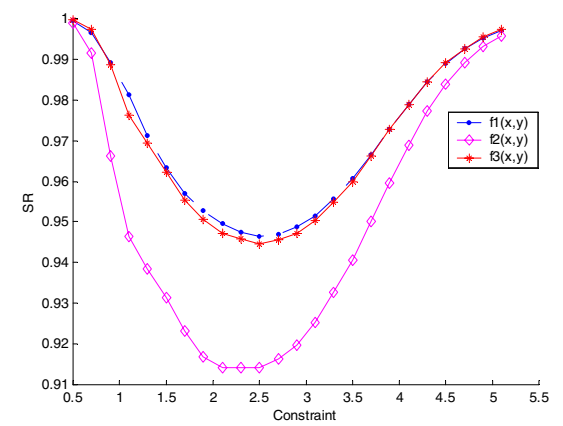

Figure 5. SR in the $7 \times 7$ mesh network.
It can be observed that, in both simulations, the QoS routing algorithm with the linear cost function achieves the highest success ratio in finding a feasible path, which conforms to our conjecture.

\section{CONCLUSIONS}

Although source routing has been extensively studied in the past, most proposed multiple constrained routing algorithms are heuristic in nature. In this paper, we have provided a theoretical framework for selecting the cost function for multiple additively constrained QoS routing. By deploying our proposed coverage ratio as the metric for evaluating the cost function, it is advisable to use a linear cost function for the approach in which the cost of a path equals the sum of the costs of all individual links comprising the path.

\section{REFERENCES}

[1] S. Chen and K. Nahsted, "An overview of quality of service routing for next-generation high-speed network: problems and solutions," IEEE Network, vol. 12, no. 6, pp 64-79, December, 1998.

[2] A. Shaikh, J. Rexford, and K.G. Shin, "Evaluating the impact of stale link state on quality-of-service routing," IEEE/ACM Transactions on Networking, vol. 9, no. 2, pp 162-176, April, 2001.

[3] R. Guerin and A. Orda, "QoS based routing in networks with inaccurate information: theory and algorithms," Proceedings of the INFOCOM'97, pp 75-83, 1997.

[4] J. Wang, W. Wang, J. Chen, and S. Chen, "A randomized QoS routing algorithm on networks with inaccurate link-state information," Proceeding of WCC-ICCT 2000, vol. 2, pp 1617-1622, 2000 .

[5] Z. Wang and J. Crowcroft, "Quality of Service routing for supporting multimedia applications," IEEE Journal on Selected Areas on Communications, vol. 14, no. 7, pp 1228-1234, September, 1996.

[6] T. Korkmaz, M. Krunz, and S. Tragoudas, "An efficient algorithms for finding a path subject to two additive constraints," Proceedings of the ACM SIGMETRICS'2000, pp 318-327, June 2000.

[7] A. Juttner, B. Szyiatovszki, I. Mecs, and Rajko, "Lagrange releaxation based method for the QoS routing problem," Proceedings of IEEE INFOCOM 2001, vol. 2, pp 859-868, 2001.

[8] L. Guo and I. Matta, "Search space reduction in QoS routing," Proceedings of $19^{\text {th }}$ IEEE International Conference on Distributed Computing Systems, pp 142-149, 1999.

[9] H. De Neve and P. van Mieghem, "A multiple quality of service routing algorithm for PNNI," Proceedings of 1998 IEEE ATM workshop, pp 324-328, 1998.

[10] T. Korkmaz and M. Krunz, "Multi-constrained optimal path selection," Proceedings of the IEEE INFOCOM 2001 Conference, pp. 834-843, April 2001. 